Manuscript submitted to Journal of Non-Newtonian Fluid Mechanics

Version: June 3, 2016

\title{
Impacts of yield-stress fluid drops on permeable mesh substrates
}

\author{
Brendan C. Blackwell, Athrey E Nadhan, Randy H. Ewoldt* \\ Department of Mechanical Science and Engineering, \\ University of Illinois at Urbana-Champaign, Urbana, IL 61801, USA
}

\begin{abstract}
Viscoplastic fluids, also known as yield-stress fluids, can stick and accumulate where they impact. Here we experimentally study conditions for open surfaces to be impermeable to impacting yield-stress fluid drops, and the dynamic conditions for these drops to permeate and coat internal aspects of a complex topography. We experimentally study drops of model yield stress fluids (Carbopol microgel particles in water) impacting open solid meshes (rigid surfaces with small, evenly spaced openings). High speed video reveals dynamics across a range of behavior, from $0 \%$ to $100 \%$ transmittance, by varying drop size, impact velocity, mesh geometry, and rheological material properties. When inertial stresses are sufficiently high compared to the yield stress, a drop can pass through a mesh, breaking into smaller fluid particles with varying shapes, sizes, and velocities in the process. In contrast, when inertial stresses are sufficiently low compared to the yield stress, a drop can stick to the mesh as though it were a solid surface, inhibited from passing through the holes by the yield stress. Layers of multiple meshes are also examined, demonstrating a range of behaviors and the ability to coat internal aspects of complex topography. Dimensional analysis is performed to characterize material transmittance and velocity of transmitted droplets as a function of dimensionless input parameters.
\end{abstract}

*Corresponding author: ewoldt@illinois.edu 


\section{Introduction}

Yield stress fluids are pervasive in industrial applications such as food processing, agriculture, grease lubrication, and manufacturing [1]. Many of these applications (including fire suppression and coating processes) involve fluid drops impacting surfaces. Significant study has already been devoted to the impact of yield stress fluid drops onto flat, dry surfaces [2-7]. Some study has been devoted to drops impacting textured surfaces [7], yet the problem of impact on permeable surfaces has not yet been addressed in the literature. As a first step into the exploration of complex topographies, our study here explores drops of yield-stress fluid impacting a flat, rigid, geometrically periodic permeable surface.

When a Newtonian fluid impacts such a surface, a small amount of fluid can adhere statically to the mesh and resist gravity due to surface tension (a balance determined by the Bond number). When the yield stress is sufficiently large, the rheological ability to maintain a static stress allows larger volume of fluid to accumulate without passing through. We show here that when the inertial stress generated by the impact is much greater than the viscous stresses and the yield stress of the fluid, the majority of the droplet can still traverse the mesh. Conversely, a permeable mesh may be effectively impermeable when the viscous and yield stresses are large enough compared to the inertial stress; in this case the entirety of a drop can be immobilized, much as though the surface were impermeable.

To explore and characterize the space between these two extremes, we design experiments varying the drop size, impact velocity, mesh geometry, and rheological material properties (Section II). We show sample images from high speed videos of representative impacts (Section III), and present data on both the percentage of material transmitted and the velocity of transmitted material (Section IV). We then perform dimensional analysis on the results, finding 
that the data are well characterized by the ratio of inertia to the sum of viscous and yield stress (Section V), similar to our prior results with drops of the same material impacting pre-coated surfaces [8].

\section{Experimental setup}

For this first exploration into this experimental space, we use a well-studied model yield stress fluid, an aqueous solution of Carbopol 940, neutralized to a $\mathrm{pH}$ of 7. Carbopol is a polymer microgel, a crosslinked polymer particle. Particles in solution form a jammed system that exhibits a yield stress when the concentration is sufficiently high. Carbopol suspensions are soft glassy materials that have a very short thixotropic restructuring time (on the order of 1 second) [9]. Figure 1 shows the steady-shear flow curves of the four concentrations of Carbopol used in this study. Data shown are sweeps from high rate to low rate, hence the measurement results in a dynamic yield stress. This is relevant for the drop impact experiments which also first expose the material to large stress at impact, which then decreases throughout the duration of the impact event. Rheological characterization was performed on a TA Instruments DHR-3 rotational rheometer using a parallel disk geometry with adhesive-backed sandpaper to prevent slip (tests were performed at multiple gaps to verify the absence of slip) [10]. Parallel disk corrections were used to compute the true shear stress from the torque measurements [11]. The curves can be reasonably approximated by fitting a two parameter Bingham model, characterizing each concentration with a yield stress and an infinite shear viscosity. Better fits can be achieved with more parameters, but in consideration of easily interpreting results we will proceed with a two parameter fit (we will discuss this further in Section IV). 


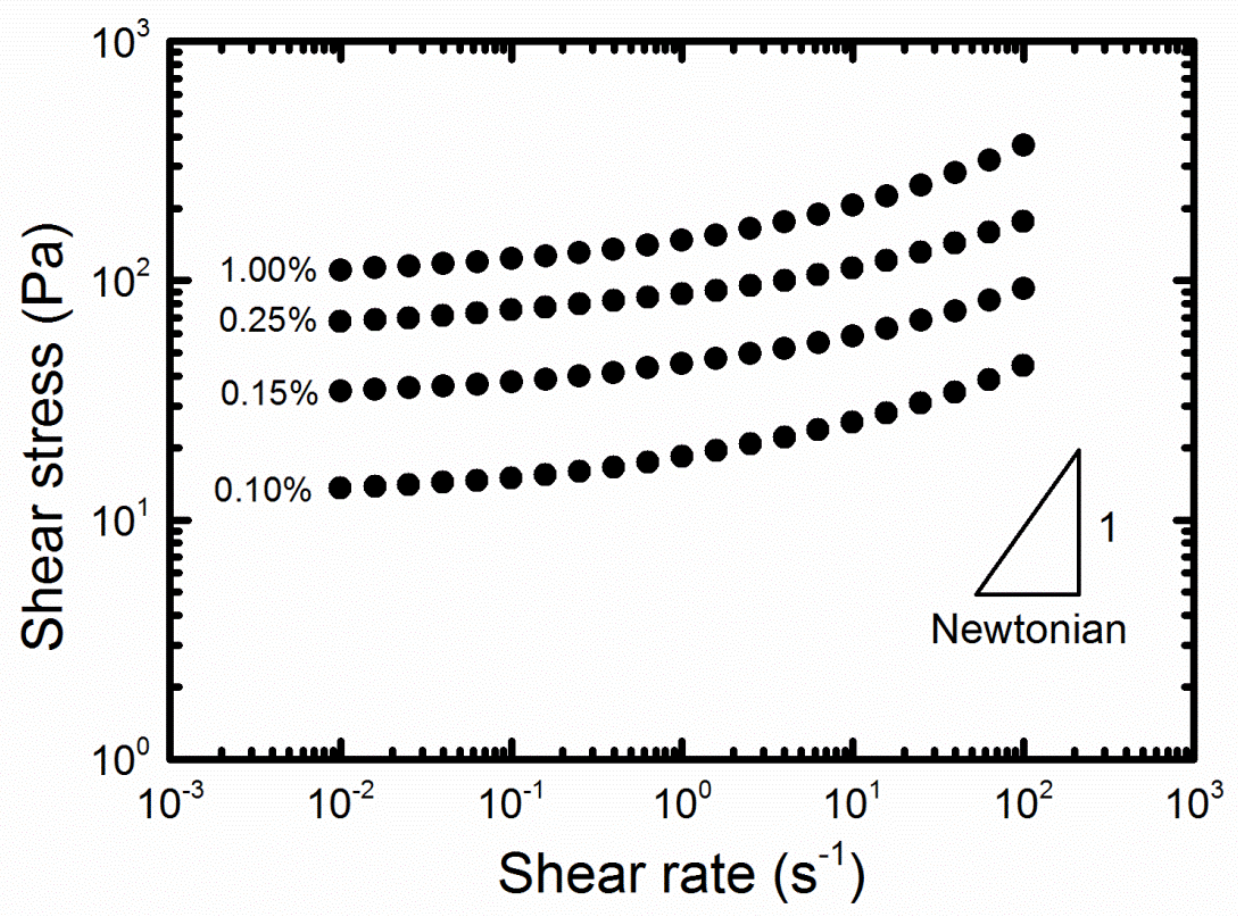

Figure 1 Steady-state flow curves for four concentrations (wt\%) of Carbopol in water (pH 7). Data are similar to Figure 1 of [8], but are measurements of different batches of the same material (agreement is very good).

Again seeking a simple case as a starting point for investigating this high-dimensional problem, geometrically periodic solid meshes are chosen as the impacting surface. First, experiments were performed using a woven aluminum wire mesh $(0.25 \mathrm{~mm}$ diameter wires spaced evenly every $1.50 \mathrm{~mm}$, as pictured and diagrammed in Figure $2 \mathrm{~b}$ ). Then, in order to examine varying geometry, experiments were performed using perforated sheets. Steel sheet was cut with a honeycomb pattern using a water jet, as pictured and diagrammed in Figure 2b, with dimensions as defined in Table 1. Three different geometries were used to compare meshes with the same hole size but different spacing, as well as the same percent open area but different hole size. The hole size in the woven mesh has some slight variation due the weave; however, the area on which drops are incident is large enough to engage several holes and produce an average 
effect, as evidenced by the variation in repeat measurements on the wire mesh being comparable to that of the cut sheet mesh.

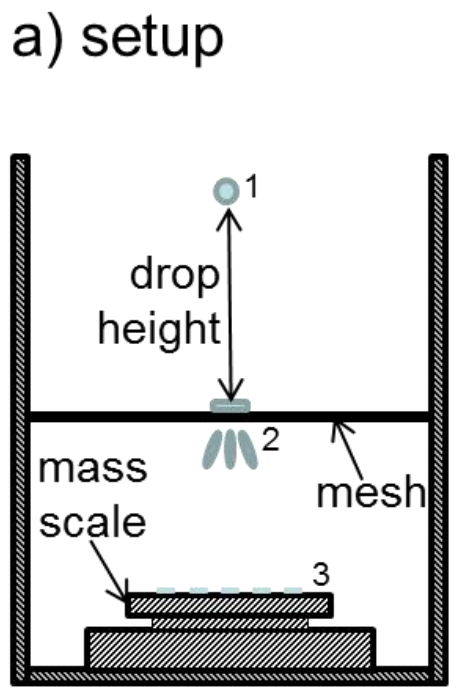

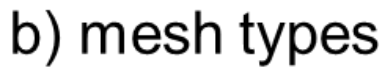

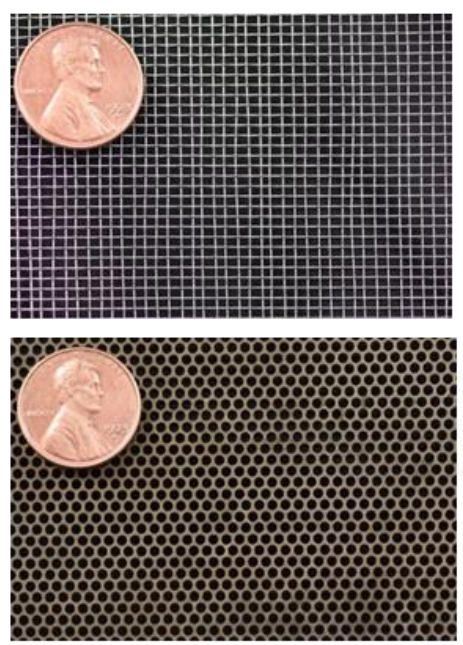

(coin for scale)
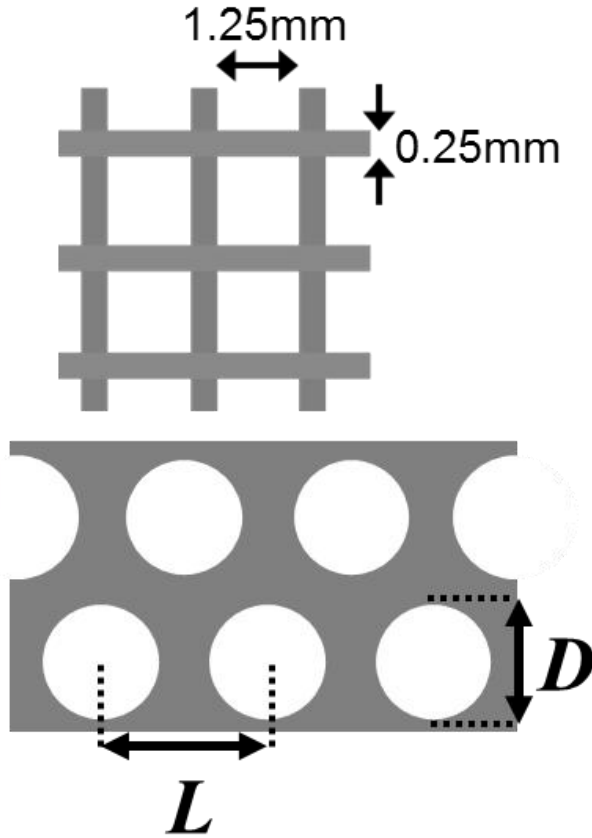

Figure 2. Experimental setup. a) Diagram of the overall setup, showing droplet at 1 initial height ,2 impact, and 3 mass collection and measurement b) photographs and dimensions of wire mesh and cut sheet (Table 1).

Table 1 Dimensions of cut sheet as drawn in Figure 2c. The percentage open area is matched between Meshes 1 and 2 but with different hole sizes. Hole size is matched between Meshes 2 and 3 but with different open area percentages.

\begin{tabular}{|l|r|r|c|}
\hline & $\boldsymbol{D}(\mathbf{m m})$ & $\boldsymbol{L}(\mathbf{m m})$ & \% Open (1- $f)$ \\
\hline Mesh 1 & 1.5 & 2.0 & 50 \\
\hline Mesh 2 & 2.0 & 2.7 & 50 \\
\hline Mesh 3 & 2.0 & 3.3 & 33 \\
\hline
\end{tabular}

Drops were dispensed into a hydrophobic concave vessel to a defined mass, then released from a defined height (which was varied to change the impacting velocity). A mass balance was placed below the impacting mesh to measure the mass of the transmitted material, as 
diagrammed in Figure 2. High speed video (using a Photron Mini-UX 100 with Nikon 105 mm macro lens) was recorded for every impact . The video recordings show the qualitative impact dynamics and were used to verify that all transmitted material was captured on the scale (spreading and splashing can allow material to escape in larger and faster drops), as well as to measure and quantify the resulting droplets created by the mesh impacts. The leading edge speed of the droplets traversing the mesh was measured by recording the time from impact to the first droplet reaching a fixed distance below the mesh. Measurements were repeated 5 times, and averaged to produce the data that are plotted in Section IV.

The range of experimental inputs (drop sizes, drop velocities, mesh sizes, material properties) is shown in Table 2, and gives ranges of standard dimensionless parameters shown in Table 3.

Table 2 Values of dimensional input parameters tested.

\begin{tabular}{|l|l|l|l|l|}
\hline Parameter & \multicolumn{4}{|l|}{ Values tested } \\
\hline Mass (g) & 0.10 & 0.30 & 1.00 & 3.00 \\
\hline Velocity (m/s) & 2.54 & 3.81 & 5.09 & 6.35 \\
\hline Concentration (\%) & 0.10 & 0.15 & 0.25 & 1.00 \\
\hline Yield stress (Pa) & & & & \\
\hline Infinite-shear viscosity (Pa.s) & 0.23 & 0.49 & 0.96 & 2.10 \\
\hline
\end{tabular}

The Bond number for all experiments ranges from about 1-5, indicating that static adhesion due to surface tension alone will be limited by gravitational forces. The Weber number for all tests is on the order of 100-1000, indicating that initial impact events will be primarily dominated by inertia, rather than surface tension. The yield stress and surface tension are also worth comparing, as two possible effects to 'stick' liquid to the meshes, and also in competition to 
determine the shape of drops. Large droplets may not be spherical [8], due to the inability of capillary stresses to deform material above a characteristic radius larger than $R_{c r i t}=\gamma / \sigma_{y}$.

Estimates of characteristic shear rates are also shown in Table 2, based on the impact velocities and mesh geometry. These largest shear rates, at impact, are outside the range of measured shear rheology in Fig. 1 by 1-2 decades. The Bingham model fits help extrapolate into this region; of course the arrested motion involves much lower shear rates that are well within the range of rheological characterization in Fig. 1.

Table 3 Ranges of standard dimensionless quantities and estimated shear rates at impact explored in this experimental space.

\begin{tabular}{|l|c|c|c|}
\hline Quantity & Definition & Minimum tested & Maximum tested \\
\hline Bond number & $\frac{\rho g D_{\text {drop }}}{\gamma / d_{\text {mesh }}}$ & 1.17 & 4.81 \\
\hline Weber number & $\frac{\rho V^{2}}{\gamma / d_{\text {mesh }}}$ & 81.3 & 868 \\
\hline Characteristic shear rate at impact & $\frac{V}{d_{\text {mesh }} / 2}$ & $1990 \mathrm{~s}^{-1}$ & $7510 \mathrm{~s}^{-1}$ \\
& & & \\
\hline
\end{tabular}

\section{Qualitative Results}

Figure 3-Figure 6 show images of drop impacts with varying additive concentration (water and three concentrations of Carbopol additive), controlled to have the same drop size $(0.4 \mathrm{~g})$ and speed $(2.5 \mathrm{~m} / \mathrm{s})$ impacting two layers of the same mesh (mesh 1 , with dimensions as defined in 
Table 1). Snapshots are shown at $7.5 \mathrm{~ms}$ increments (from $2.5 \mathrm{~ms}$ before impact to $50 \mathrm{~ms}$ after impact).

Important qualitative features to visualize include the extent to which a drop penetrates through the meshes, the amount of material that 'sticks' on each mesh (which we quantify), whether accumulated material is on top of or protruding through the mesh (not quantified here), the resulting drop size distribution (not quantified here), and the velocity of droplets that have passed through the mesh (which we quantify).
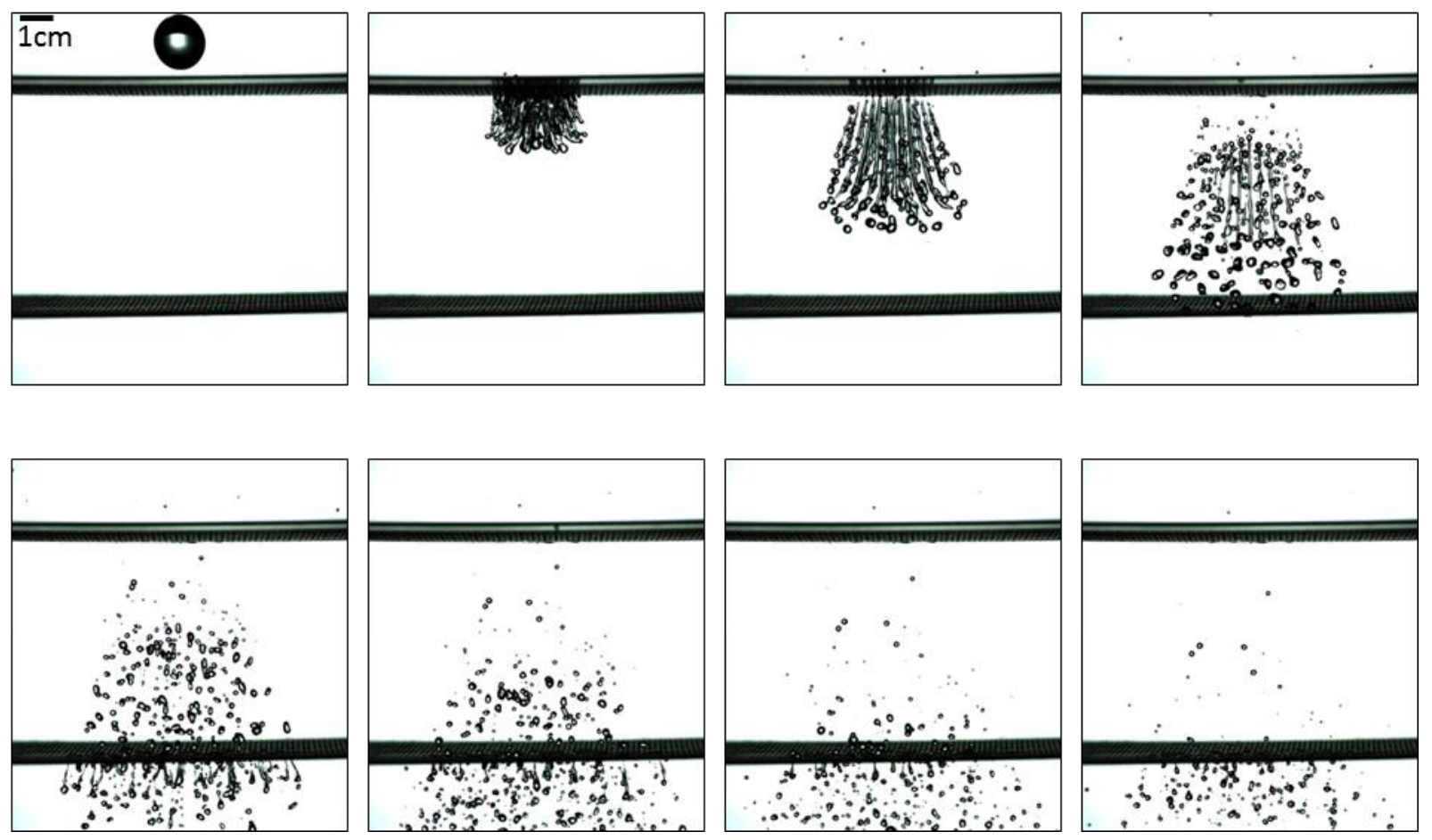

Figure 3 Water drop $(0.4 \mathrm{~g})$ impacting mesh 1 at $2.5 \mathrm{~m} / \mathrm{s}$. Time increment between images is $7.5 \mathrm{~ms}$. (See Supplemental Information for full video)

Figure 3 shows an example water drop impact. In this event the threads that result from the drop pushing through the first mesh quickly breakup into droplets due to surface tension (socalled Rayleigh-Plateau instability), showing almost exclusively spherical droplets within 20ms of the initial impact. As the material passes through the second mesh, the initial droplets are broken into even smaller droplets, and the velocities of the individual droplets become more 
widely varied. Nearly all (over 95\%) of the mass is transmitted (as measured at the mass balance below the meshes); surface tension can adhere only a small mass of water to the meshes.
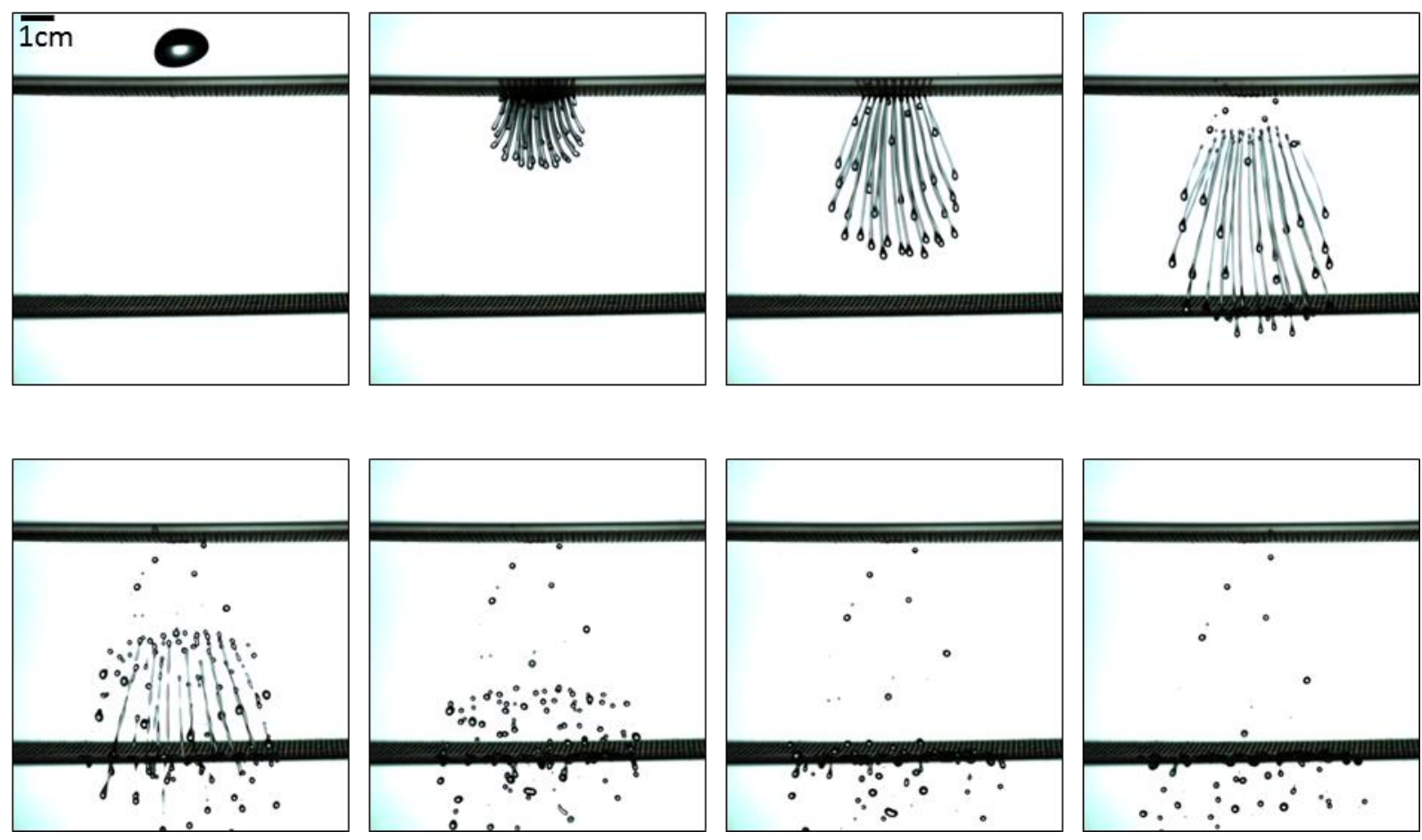

Figure 4 A $0.4 \mathrm{~g}$ drop of $0.10 \%$ Carbopol in water (dynamic yield stress $\sigma_{y}=13 \mathrm{~Pa}$ ) impacting mesh 1 at $2.5 \mathrm{~m} / \mathrm{s}$. Time increment between images is $7.5 \mathrm{~ms}$. (See Supplemental Information for full video)

Figure 4 shows an example yield-stress fluid impact in which inertia is greater than dissipative forces (a drop of $0.10 \%$ Carbopol with the same size and speed as the water drop in Figure 3). In contrast to the water drop impact, the jets that form as the material emerges from the first mesh survive longer before breaking into droplets, as the material has a higher viscosity than water. A lower velocity of the final droplets exiting the mesh matrix is also observed, which we attribute to the higher viscosity. The yield stress is small enough such that the final droplets are still quasi-spherical, as capillary forces are strong enough to overpower the yield stress and evolve the shape. The yield stress does allow more material to adhere to each mesh, but with the yield stress being comparable to the capillary stresses, the additional amount is on the same order as the capillary adhesion (which is still a small percentage of the total mass). 

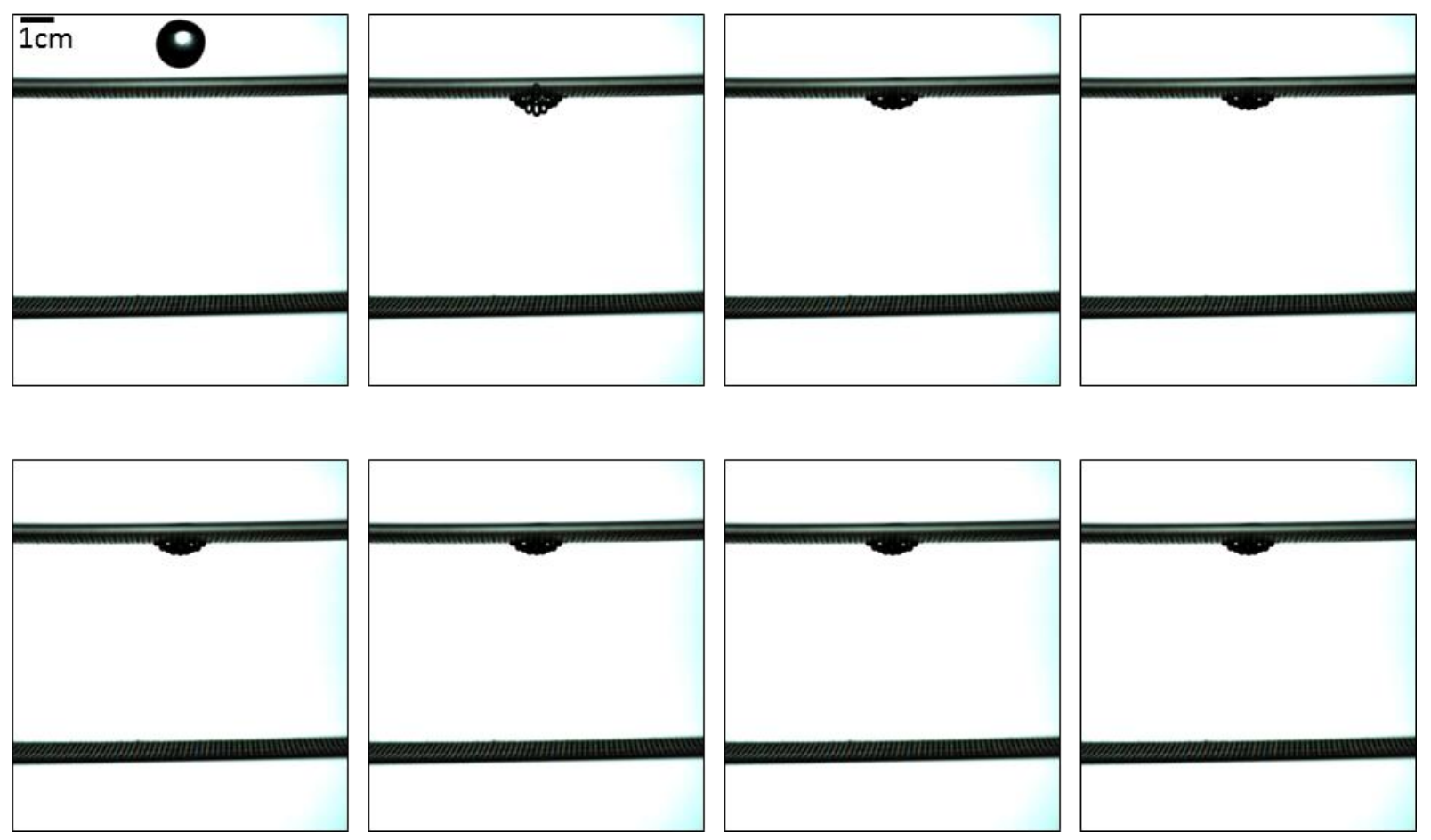

Figure 5 A $0.4 \mathrm{~g}$ drop of $0.25 \%$ carbopol (dynamic yield stress $\sigma_{y}=64 \mathrm{~Pa}$ ) impacting mesh 1 at $2.5 \mathrm{~m} / \mathrm{s}$. Time increment between images is $7.5 \mathrm{~ms}$. (See Supplemental Information for full video)

Figure 5 shows an example yield-stress fluid impact in which the yield stress is greater than inertia (a drop of $0.25 \%$ Carbopol with the same size and speed as the drops in Figure 3 and Figure 4). In this event, the drop is unable to pass through the first mesh at all. While the drop remains one contiguous mass, some material does squeeze through the holes. However, the tension in the strands that extrude through is not high enough to break them off. The material does show some elasticity, as the strands stretch (as seen in the second panel) and retract (as seen in the third panel). 

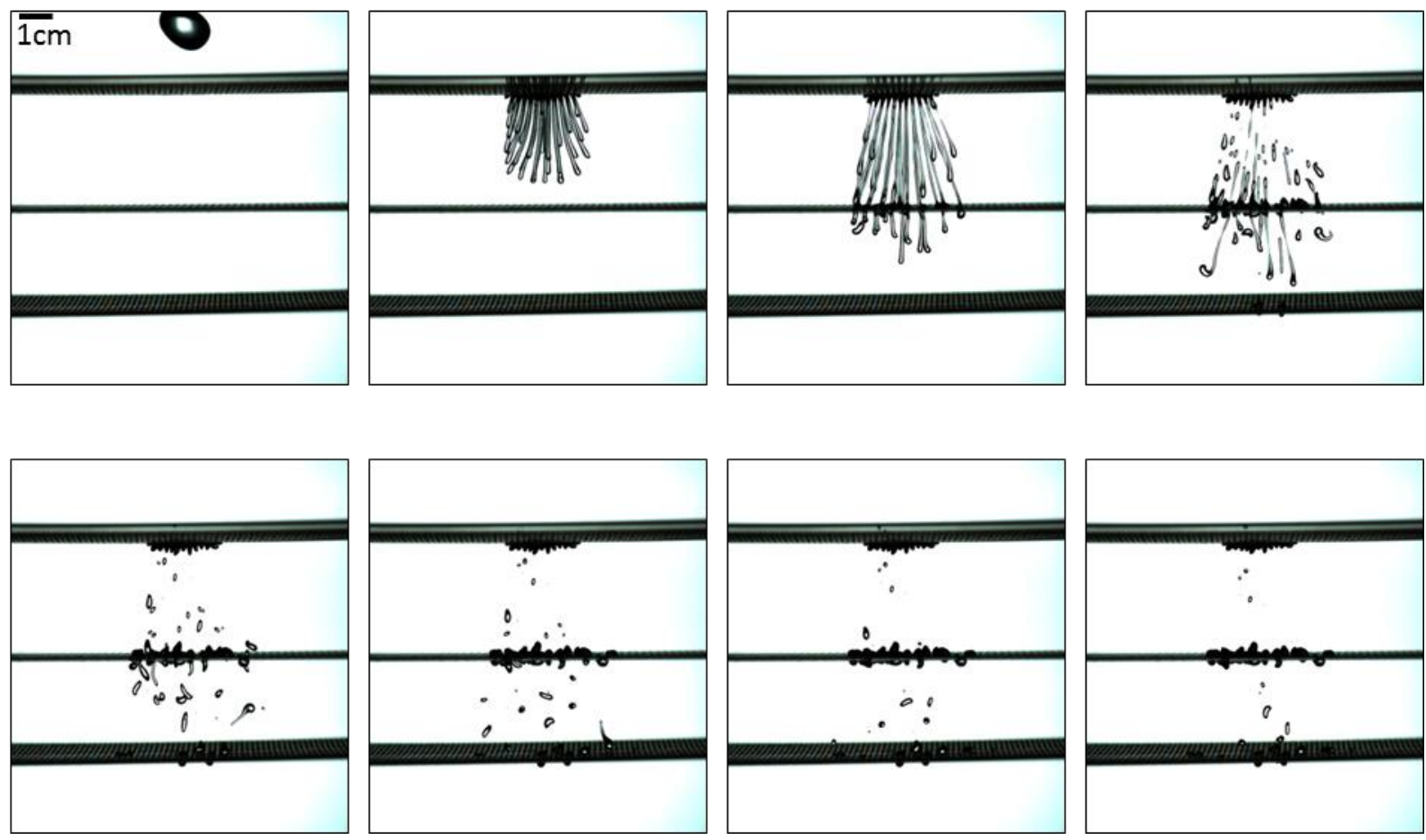

Figure 6 A $0.4 \mathrm{~g}$ drop of $0.15 \%$ carbopol (dynamic yield stress $\sigma_{y}=33 \mathrm{~Pa}$ ) impacting mesh 1 at $2.5 \mathrm{~m} / \mathrm{s}$. Time increment between images is $7.5 \mathrm{~ms}$. (See Supplemental Information for full video)

Figure 6 shows an intermediate condition in which the yield and inertial stresses are comparable (a drop of 0.15\% Carbopol with the same size and speed as the drops in Figure 3Figure 5). A third mesh is included to demonstrate coating of internal aspects of complex topography. Similar to the impact of $0.10 \%$ Carbopol, the strands emerging from the first mesh evolve more slowly into droplets than in the water impact in Figure 3. In contrast, the yield stress in this case is large enough such that the resulting droplets can stably maintain non-spherical shapes. In this event material is deposited at all three layers of the mesh matrix, showing the interesting midpoint between the transmission extreme of Figure $\mathbf{4}$ and the adherence extreme of

\section{Figure 5.}

These qualitative results demonstrate unique physics of yield stress fluids, which can coat complex topography, including 'internal' topography as shown with the multiple coated meshes of Figure 6. The materials stick, and do not drip, even over long observational timescales. To 
more fully characterize this behavior, we proceed to quantify two key features across a wide parameter space of drop size, drop velocity, and yield stress: (i) the amount of mass transmitted through a mesh (or series of meshes) and (ii) the velocity of transmitted material leaving the mesh, from image analysis of high speed videos.

\section{Quantitative Results}

We quantify how much material 'sticks to' versus 'passes through' a mesh by the percentage of mass transmitted, as measured by a mass balance below the mesh. Figure 7 shows material transmittance data through a single wire mesh (Figure 2b) as a function of drop mass, drop velocity, and Carbopol concentration. The full range of behavior from $0 \%$ to nearly $100 \%$ transmittance is covered in the experiments, as indicated by the color bar. Each subplot varies drop mass (on the abscissa) and impact velocity (on the ordinate), while concentration varies from subplot to subplot. Values of mass, velocity, and concentration are those shown in Table 2. It is worth noting that droplet shape varies slightly from test to test, as the drops studied are above the capillary length and can therefore be steadily non-spherical due to their yield stress. The small spread between repeat measurements indicates that this does not substantially affect the data (all transmittance data shown is the average of five measurements). Material transmittance increases monotonically with increasing velocity and mass (higher drop momentum), and decreasing concentration (lower yield stress). 


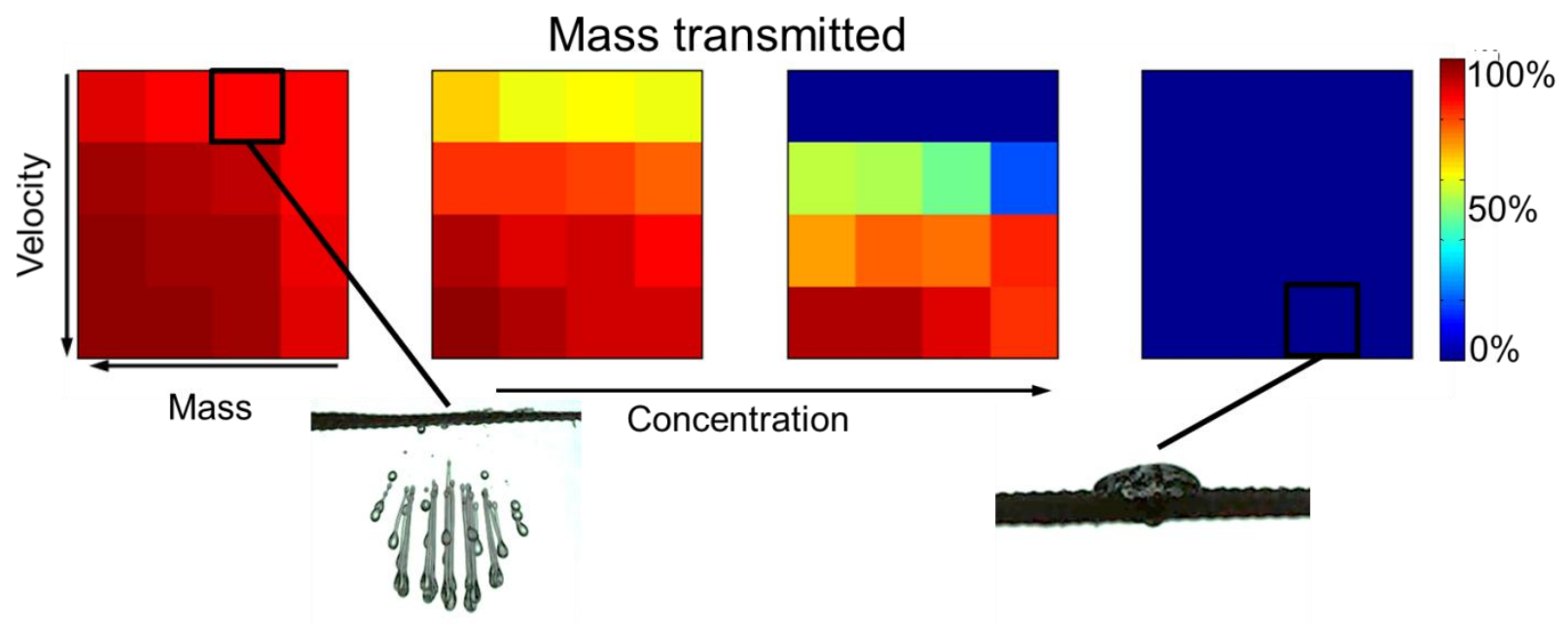

Figure 7 Percent of incident material that is transmitted through a single wire mesh for varying drop mass, velocity, and material concentration. The full range of behavior from $0 \%$ to nearly $100 \%$ transmittance is covered in the experiments, as indicated by the color bar.

The transmittance shows monotonic trends with respect to all three input variables: higher velocity, larger mass, and lower concentration all uniformly result in a higher percentage of the droplet passing through the mesh. The transmitted quantity shows low sensitivity to changing values of the inputs when the inputs are at their extreme values, with a sharp transition at intermediate values. The reason for the sharp transition from zero to finite transmission (the smallest nonzero data points are roughly 50\%) is explained by the dynamics of the strands extruding through the mesh, roughly as observed in the second panel of Figure 6. When transmittance is zero, the tension is not high enough to break the strands. Transmittance quantity quickly increases at the point where inertia overcomes the yield stress; the stress in the strand is maximum at the top, hence the entire strand breaks off. By the time the balance is sufficient to break the strands the extruded material constitutes a significant portion of the mass of the drop, hence the transmittance quickly jumps from $0 \%$ to $50 \%$ or more. This effect will be seen as sharp upturns in the transmittance data in Figure 10 and Figure 14. 


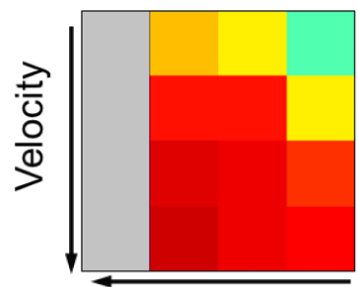

Mass

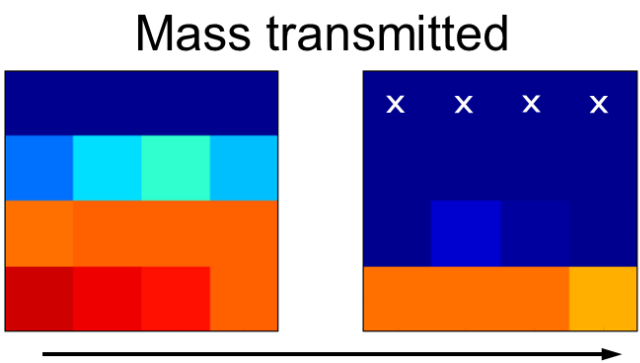

Concentration

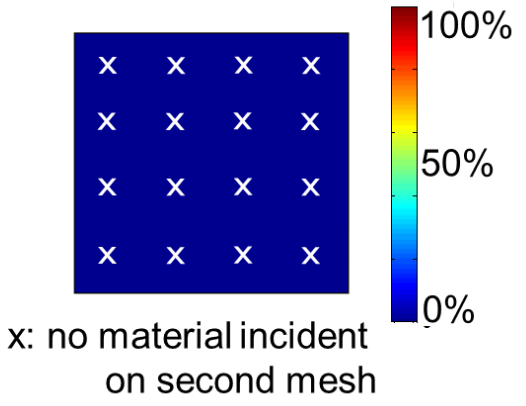

on second mesh

Figure 8 Percent of incident material that is transmitted through two wire meshes for varying drop mass, velocity, and material concentration. White x's indicate that all material was captured by the first mesh. Grey data points represent data not collected due to excessive splashing of transmitted material which did not rest on the mass balance.

Figure 8 shows transmittance data through two wire meshes (spaced $5 \mathrm{~cm}$ apart, similar to Figures 3-6), for the same experimental conditions arranged identically to the data in Figure 7. As with one mesh, the trends with two meshes are monotonic with respect to all three input variables. However, the transmittance does not show as sharp of a transition from $0 \%$ to finite values. The smoother change is due to the polydispersity in size of the secondary droplets created by the first mesh which impact the second mesh. The tumbling, irregular shaped, variously sized droplets allow for smaller finite transmittance through the second mesh than are produced by the initial drops. Figures 7-8 show clear general trends in a high-dimensional space; to further explore the physics and increase the utility, we proceed to examine the data as a function of dimensionless groups.

\section{Dimensionless groups}

The goal is to reduce the dimensionality of the space as much as possible, ideally showing the output data as a well-behaved function of a single parameter. While the transmittance monotonically trends with each input variable when the values of the other inputs are fixed, the data does not collapse well as a function of a single variable when all inputs are free. Figure 9 
demonstrates this, plotting transmittance against velocity for all 64 data points shown in Figure 7.

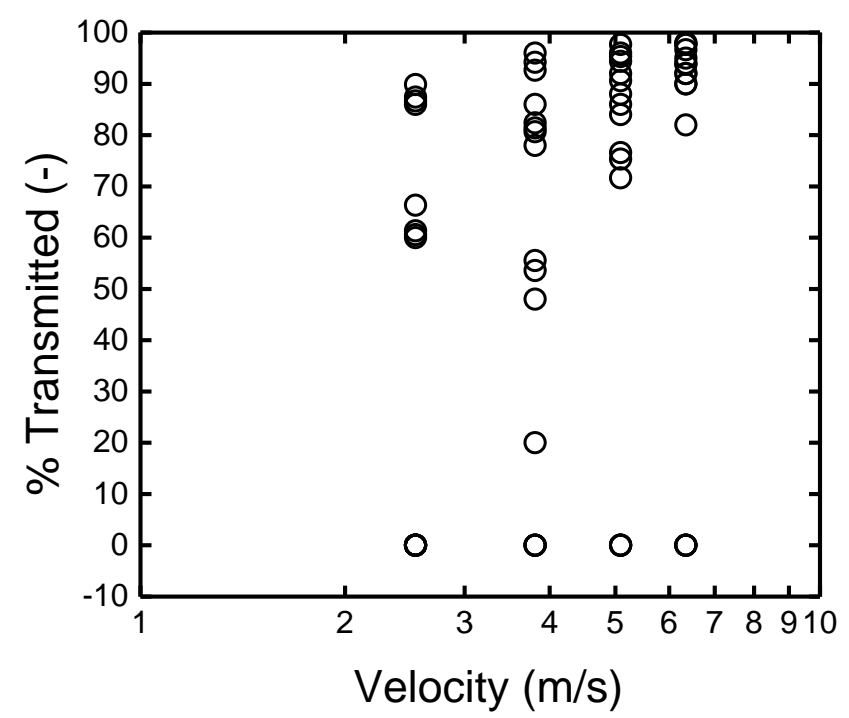

Figure 9 Material transmittance plotted against velocity for a single wire mesh.

The plot does show a general increasing trend, however the spread is very large. Better description of the transmittance can be achieved by a properly constructed dimensionless group. As the physics of adherence is dominated by the balance of inertia and the yield stress, we first plot transmittance as a function of $\rho V^{2} / \sigma_{y}$, as shown in Figure 10. This framing collapses the data much better than any single input variable. 


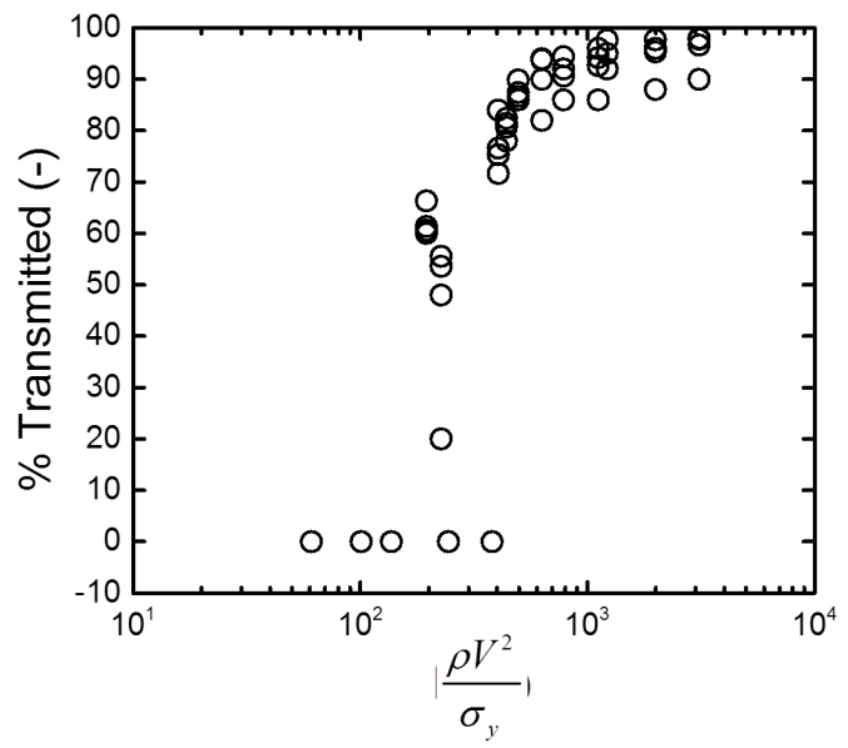

Figure 10 Material transmittance plotted against the ratio of inertia to yield stress for a single wire mesh.

Similar results can be shown for the leading edge velocity of material that has traversed the mesh. The wide spread of data as a function of a single variable is demonstrated by Figure 11, which shows exit velocity normalized by entrance velocity plotted against yield stress. The expected general decreasing trend is captured, but the value of the individual input is not predictive of the output with any precision. 


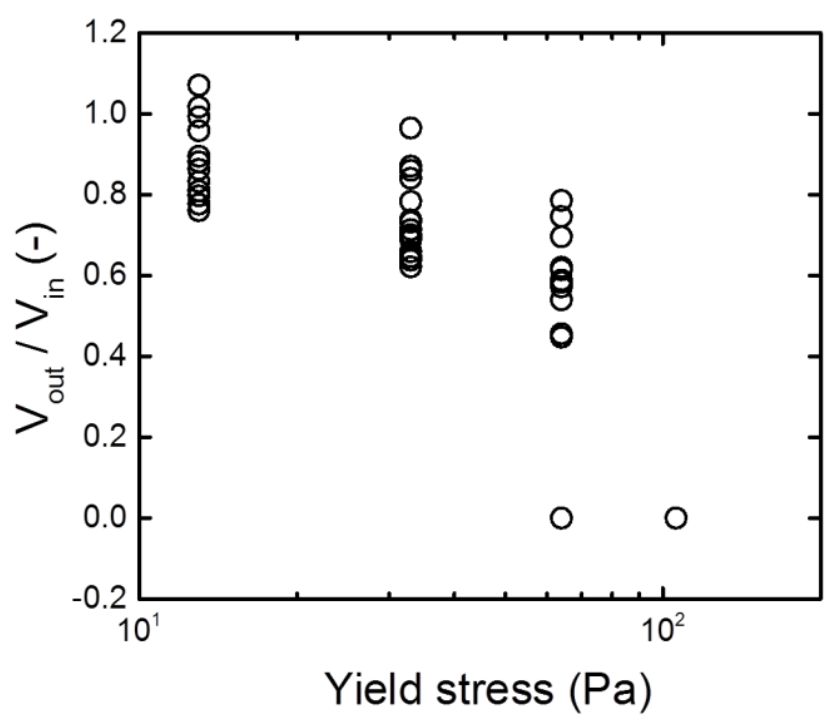

Figure 11 Normalized exit velocity plotted against yield stress for a single wire mesh.

In contrast, we find that the exit velocity is much more reliably related to the quantity $\rho V d_{\text {mesh }} / \eta_{\infty}$, a comparison of inertial stress to high shear rate viscous stress as plotted in Figure 12. The full range from $0-1$ is covered by the experiments. The exit velocity is nearly equal to the impact velocity when this ratio is $\frac{\rho V d_{\text {mesh }}}{\eta_{\infty}} \sim 30$ (Note that as velocity is calculated as the average velocity of the leading droplet over a defined distance after exiting the mesh, gravitational acceleration does slightly increase this measurement; however, this contribution is small in magnitude compared to the overall signal.)

The different dimensionless groups for transmittance (Figure 10) and exit velocity (Figure 12) can be understood by viewing the exit velocity as a high velocity (high shear rate) process of slowing down a droplet, versus the transmittance which is a low velocity (low shear rate) process associated with capturing material on a mesh, fully stopping its motion, and resisting a static gravitational load. 


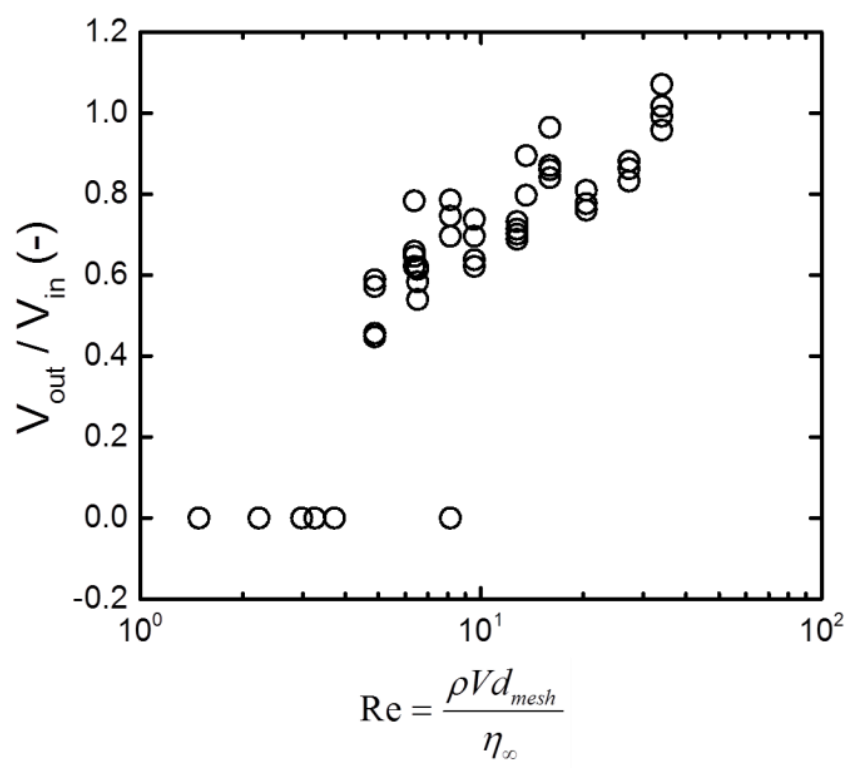

Figure 12 Normalized exit velocity plotted against Reynolds number for a single wire mesh.

The mesh geometry should influence mass transmittance and droplet exit velocity. The characteristic size of the open spaces is involved in the dimensionless group $\rho V d_{m e s h} / \eta_{\infty}$, though not $\rho V^{2} / \sigma_{y}$, and neither group yet involves another mesh parameter: the area fraction of solid material. To test the robustness of the dimensionless group for transmittance (and develop an improved but more complex dimensionless group), Figure 13 plots transmittance against $\rho V^{2} / \sigma_{y}$ for each of the three cut sheet meshes, which vary hole size and fill area (as drawn in Figure 2c with dimensions as defined in Table 1). 


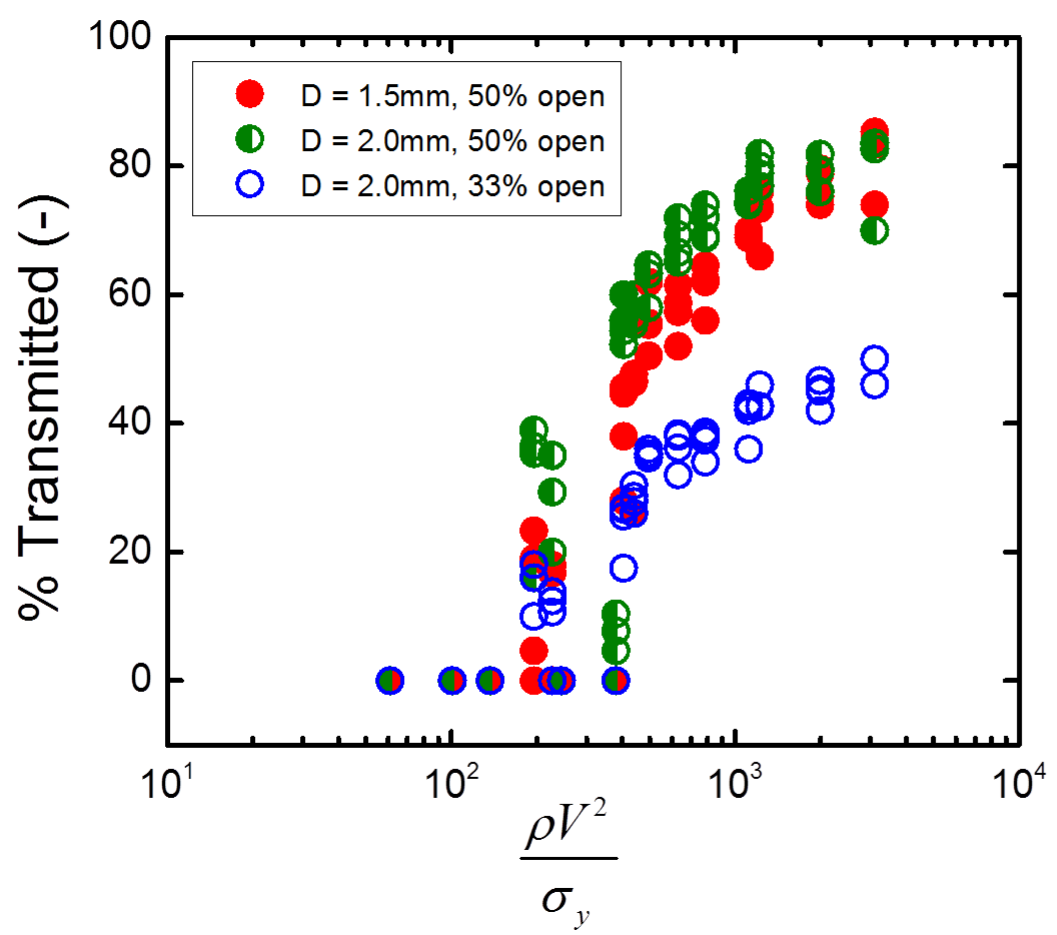

Figure 13 Material transmittance plotted against the ratio of inertia to yield stress for three different mesh geometries.

For each individual geometry the data collapse nicely in Figure 13 . However, each of the three curves traces its own path through the parameter space. Therefore while the parameter $\rho V^{2} / \sigma_{y}$ is useful for a fixed geometry, it fails to capture all of the physics involved. The characteristic opening size (diameter) and fill area should be taken into account. Prior work on drop impacts of the same material [8] found good reduction of the data using the ratio of inertia to the sum of viscous and yield stresses. Here we find it useful to modify this parameter by the fraction of solid area of the mesh, $f$, arriving at the parameter (named 'IF' as the ratio of inertia to a flow stress)

$$
\mathrm{IF}=\frac{\rho V^{2}}{f \cdot\left(\sigma_{y}+\eta_{\infty} V / D\right)}
$$


Additionally, we can normalize the measured percent of transmitted material by the percent open area of the mesh; plotting it against IF produces the plot shown in Figure 14, which collapses the transmittance data better than Figure 13.

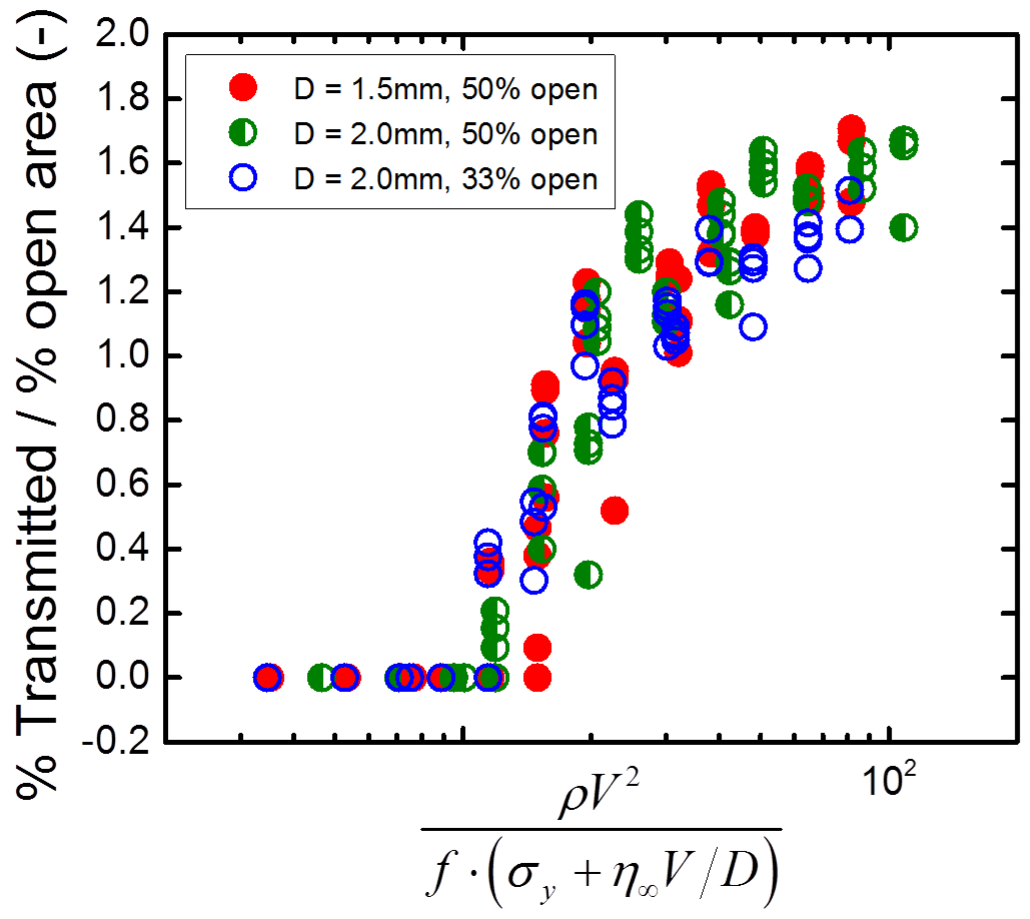

Figure 14 Normalized material transmittance plotted against IF for all three varied mesh geometries. Error bars omitted for legibility because they are smaller than the symbol size.

Figure 14 nicely collapses the data for all three meshes onto a single curve with comparably small spread. Across all observed values of drop size, drop velocity, rheological properties, mesh hole size, and mesh hole spacing, the value of IF is predictive of the percentage of material transmitted through the mesh. An interesting feature of this plot is that transmittance sharply increases from zero to moderate values over a small range of IF, with very few data points at small but nonzero values. This occurs because the transition from zero to finite transmittance involves long strands of material extruding through and trying to adhere to the mesh (see the 
second panel of Figure 5). With increasing IF, the strands become longer and longer until inertia overcomes the yield and dissipative stresses, at which point the strands break. Because the highest stress is at the interface with the mesh, the entire strand (which by now represents a nontrivial portion of the drop mass) breaks off. This is why the transition from zero to moderate transmittance values is so abrupt.

Figure 15 shows the observed exit velocity against the same parameter. Although the velocity does not collapse as tightly as the transmittance, the trend is still significantly more effectively characterized than by a single input variable.

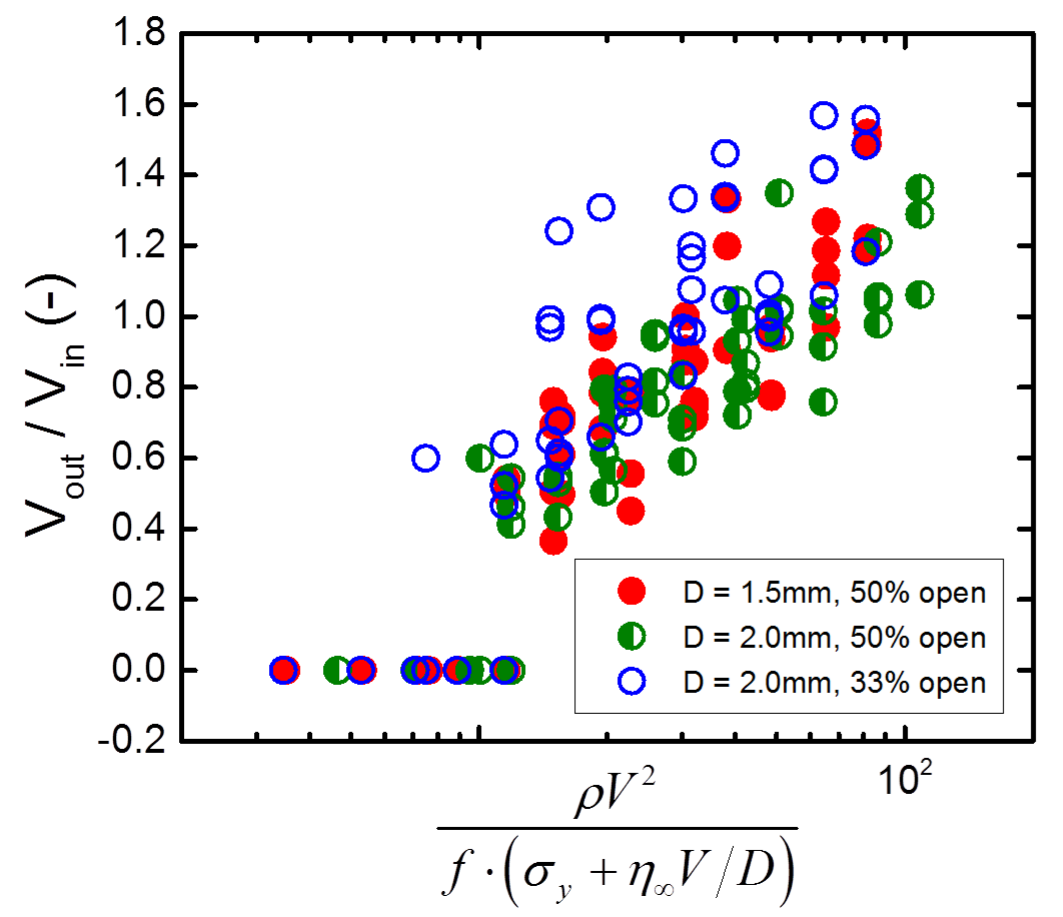

Figure 15 Normalized leading edge exit velocity plotted against IF for all three varied mesh geometries. 


\section{Conclusions}

Here we report the curious phenomena of yield-stress fluid drops at high Weber number that can be completely halted by thin permeable substrates (meshes). Yield-stress fluid droplets can stick to an open solid mesh without any dripping through, as though the substrate was impermeable. i.e. permeable surfaces may be impermeable to viscoplastic droplets due to the yield stress of the material. A drop can pass through with sufficient impact momentum. We have documented a data set covering a range from $0 \%$ to nearly $100 \%$ transmittance through various mesh geometries, by varying drop size, drop impact velocity, and rheological properties.

We demonstrate that the transition from transmittance to adherence occurs with decreasing input momentum (decreasing velocity and droplet size) and increasing dissipation (increasing concentration). We consider dimensionless groups to reduce the data set, after demonstrating that individual input parameters do not adequately characterize the problem. By constructing a dimensionless group to properly incorporate yield stress effects, viscous effects, inertia, and mesh geometry, we show that the ratio of the inertial forces to flow forces, calculated as $\frac{\rho V^{2}}{f\left(\sigma_{y}+\eta_{\infty} V / D\right)}$, is predictive of both the percentage of material transmitted and the leading edge velocity of exiting material. This single parameter reduces the dimensionality of the space one must examine to determine droplet dynamics, lends insight into the physics of the impact problem, and potentially allows for extrapolation of these results to dynamically and geometrically similar situations beyond the explicit parameter values explored here. Of course, many open questions remain, and this experimental dataset is only a start to understanding viscoplastic fluid droplet impacts on non-trivial geometries. One can easily recognize a vast range of different experimental conditions available, including other geometries, surface 
chemistries, or changing the relative direction of gravitational forces compared to substrate orientation (e.g. sticking on a wall or ceiling).

The current experimental data set stands as an opportunity and challenge for further theoretical understanding and computational prediction. We anticipate important questions related to constitutive model selection, in particular on the choice to include viscoelasticity and/or thixotropy in the models. Constitutive models quickly become complex when adding viscoelastic or thixotropic phenomena, and even more complex with both! The essential ingredients for prediction therefore stand as an open question for modeling and simulation of the data set described here. Experimental characterization of thixotropy is non-trivial. Moreover, the impact timescales are short, on the order of 3-30 ms, which is at the edge or below typical experimental limits of shear rheometers. Thus, our study also motivates the need for advanced rheological characterization techniques that probe transient nonlinear effects in viscoplastic liquids $[12,13]$.

\section{Acknowledgements}

This work was supported by the National Science Foundation under Grant No. CBET-1351342. R.H.E. is grateful to the organizers and participants at the workshop "Viscoplastic Fluids: From Theory to Application VI" in Banff, Canada, October 2015, for stimulating discussions.

\section{References}

[1] N.J. Balmforth, I. a. Frigaard, G. Ovarlez, Yielding to Stress: Recent Developments in Viscoplastic Fluid Mechanics, Annual Review of Fluid Mechanics. 46 (2014) 121-146. 
[2] S. Nigen, Experimental investigation fo the impact of an (apparent) yield-stress material, Atomization and Sprays. 15 (2005) 103-117.

[3] L.-H. Luu, Y. Forterre, Drop impact of yield-stress fluids, Journal of Fluid Mechanics. 632 (2009) 301-327.

[4] G. German, V. Bertola, Impact of shear-thinning and yield-stress drops on solid substrates., Journal of Physics: Condensed Matter. 21 (2009) 375111.

[5] S. Rahimi, D. Weihs, Gelled fuel simulant droplet impact onto a solid surface, Propellants, Explosives, Pyrotechnics. 36 (2011) 273-281.

[6] E. Kim, J. Baek, Numerical study of the parameters governing the impact dynamics of yield-stress fluid droplets on a solid surface, Journal of Non-Newtonian Fluid Mechanics. 173-174 (2012) 62-71.

[7] M. Guémas, Á.G. Marín, D. Lohse, Drop impact experiments of non-Newtonian liquids on micro-structured surfaces, Soft Matter. 8 (2012) 10725.

[8] B.C. Blackwell, M.E. Deetjen, J.E. Gaudio, R.H. Ewoldt, Sticking and splashing in yieldstress fluid drop impacts on coated surfaces, Physics of Fluids. 27 (2015) 043101.

[9] J. Labanda, P. Marco, J. Llorens, Rheological model to predict the thixotropic behaviour of colloidal dispersions, Colloids and Surfaces A: Physicochemical and Engineering Aspects. 249 (2004) 123-126.

[10] R.H. Ewoldt, M.T. Johnston, L.M. Caretta, Experimental challenges of shear rheology: how to avoid bad data, in: S. Spagnolie (Ed.), Complex Fluids in Biological Systems, Springer, 2015.

[11] C.W. Macosko, Rheology principles, measurements, and applications, Wiley, New York, 1994.

[12] B.C. Blackwell, R.H. Ewoldt, A simple thixotropic-viscoelastic constitutive model produces unique signatures in large-amplitude oscillatory shear (LAOS), Journal of NonNewtonian Fluid Mechanics. 208-209 (2014) 27-41.

[13] B.C. Blackwell, R.H. Ewoldt, Non-integer asymptotic scaling of a thixotropic-viscoelastic model in large-amplitude oscillatory shear, Journal of Non-Newtonian Fluid Mechanics. 227 (2016) 80-89. 